\title{
LE ELEZIONI NELLA CECOSLOVACCHIA DEMOCRATICA (1990-1992)
}

\author{
di Anna Campione
}

\section{Il sistema di rappresentanza prima del 1989}

Nei decenni precedenti l'instaurazione dei regimi comunisti nessuno dei sistemi politici dell'Europa centro-orientale, ad eccezione della Cecoslovacchia e della Germania, aveva sviluppato strutture partitiche relativamente forti, specie se confrontate con i sistemi democratici dell'Europa occidentale. Dopo la breve parentesi post-bellica di competizione «pluralistica» gli organi rappresentativi e le elezioni stesse erano stati trasformati in strumenti funzionali al dominio del partito unico. Benché il parlamento costituisse secondo le costituzioni di quei paesi l'organo più autorevole dello stato, le stesse avevano finito per recepire il principio (stabilito dalla costituzione sovietica del 1977) del partito comunista come «forza guida della società» (Ulc 1982)

Le elezioni (di conferma più che di scelta) non producevano reali mutamenti nell'ambito del governo e della classe politica: ai cittadini era negato il diritto di scegliere liberamente $i$ propri rappresentanti. e gli stessi deputati eletti non erano dotati di un potere politico reale. Nei parlamenti comunisti il dibattito, tranne brevi periodi come quello della Primavera di Praga, era praticamente assente e l'approvazione unanime delle leggi costituiva la regola (Holmes 1986). Nella Cecoslovacchia comunista le elezioni si svolgevano ogni quattro anni e cominciavano con una campagna elettorale suddivisa in due parti: una di «preparazione» (compilazione dei registri elettorali, definizione delle circoscrizioni, formazione dei comitati elettorali, designazione dei candidati proposti dal Fronte Nazionale', pubblica-

${ }^{1}$ Il Fronte Nazionale era l'istituzione politica, creata dal regime comunista, che riuniva tutti i partiti politici, le organizzazioni sociali e i gruppi di interesse formalmen- 
zione delle liste) e una di «esecuzione», durante la quale si svolgevano i comizi e le riunioni elettorali e venivano presentati all'elettorato i candidati delle singole circoscrizioni (Hazan 1985). Le elezioni, che erano di tipo plebiscitario (Pravda 1986), prevedevano un candidato unico per ogni singolo seggio, ed una sola piattaforma elettorale elaborata dal Fronte Nazionale; solo negli ultimi anni del regime la legge elettorale aveva subito alcune modifiche tendenti ad introdurre candidature multiple (Lovenduski e Woodall 1987, 289). L'elettore aveva la facoltà di cancellare dalla scheda il nome del candidato ufficiale, ma, dovendo in questo caso usare la cabina elettorale, il comportamento «deviante» si palesava facilmente con prevedibili conseguenze sul piano della effettiva libertà di voto. Di conseguenza i risultati elettorali non costituivano mai un'incognita per i candidati del partito comunista e dei partiti «satellite»².

Se è vero che i parlamenti comunisti non avevano un potere politico reale e le elezioni erano di fatto procedimenti fittizi quanto a potere di scelta, è altrettanto vero che le leadership comuniste se ne servivano per dar prova dell'identificazione esistente tra regime e popolazione, e per garantire ogni quattro anni il turnover di una parte dei deputati. In un determinato numero di casi, infatti, l'affidamento di un incarico parlamentare costituiva una sorta di onore o premio per «remunerare» attività di militanza, agitazione e propaganda o alti indici di produttività nel lavoro. La designazione dei candidati da parte del Fronte Nazionale avveniva secondo il criterio della rappresentanza per quote, un modello di rappresentanza di tipo sociologico e simbolico sull'esempio di quello utilizzato in URSS, e giudicato dalla dottrina ufficiale sovietica maggiormente «rappresentativo» di quello «borghese», tipico delle democrazie occidentali, basato sul principio politico-elettivo (Cappelli 1992). Sulla base del concetto sociologico di rappresentanza (Pitkin 1967),

te ammessi ad operare dal regime stesso. Il ruolo istituzionale del Fronte era quello di elaborare un'unica piattaforma politica, con il coinvolgimento, nella fase della discussione, di tutti i partiti politici e le organizzazioni che esso raggruppava. La sua attività politica è stata interrotta il 7 febbraio del 1990. Cfr. Radio Praga, 26 luglio 1989, riportato in Jiri Pehe (1990), Activities of the National Front Suspended, in «Radio Free Europe», I, n. 9.

${ }^{2}$ La Cecoslovacchia, insieme con la Bulgaria, RDT e Polonia, aveva adottato un sistema formalmente pluripartitico. Oltre al partito comunista, infatti, operavano il Partito Socialista Cecoslovacco, il Partito Popolare Cecoslovacco, il Partito della Ricostruzione Slovacco e il Partito della Libertà Slovacco. 
gli organismi rappresentativi dovevano riflettere proporzionalmente i gruppi socio-occupazionali presenti nella comunità, in modo da riprodurre, per così dire, il macrocosmo sociale. La necessità di affermare simbolicamente la funzione guida e il ruolo centrale attribuito al lavoro manuale e alla classe operaia dalla dottrina marxista-leninista, faceva sì che quest'ultima venisse sovrarappresentata in tutti i corpi elettivi (Di Leo 1981).

\section{Verso un nuovo modello di rappresentanza}

Così come gli eventi innescati in URSS nella seconda metà degli anni '80 e venuti a maturazione nel 1989 hanno portato alla crisi e poi al tracollo del modello sovietico in tutta l'Europa orientale, anche in Cecoslovacchia la «rivoluzione di velluto» dell'autunno 1989 è stata stimolata e resa possibile dai mutamenti verificatisi nel regime sovietico sotto la leadership di Gorbaciov.

La scarsa legittimazione di cui godeva ormai il regime comunista, la cristallizzazione e il mancato ricambio non solo dell'élite politica, $\mathrm{ma}$ in generale della classe dirigente, sono state tra le cause fondamentali del «crollo» ${ }^{3}$ del regime cecoslovacco. L'obiettivo dei nuovi riformatori, a differenza che nella Primavera del 1968 , non è stata più la riforma del regime esistente, ma la transizione verso un sistema di democrazia parlamentare di tipo occidentale.

Diversamente che negli altri paesi dell'Europa orientale, il Partito Comunista cecoslovacco non ha svolto un ruolo di primo piano nell'organizzazione delle prime libere elezioni parlamentari del giugno 1990, anche se esso, assieme ai cinque partiti presenti nel governo federale ed ai due piccoli partiti slovacchi, ha preso parte ai negoziati sulle riforme politiche. È apparso allora subito chiaro che i protagonisti principali del processo di transizione sarebbero stati i gruppi di opposizione ed, in special modo, due movimenti politici, il Forum Civico $(F C)$ e l'omologo movimento slovacco i Cittadini contro la Violenza $(\mathrm{CcV})$, composti in maggioranza da intellettuali, scrittori, attori, ricercatori, insegnanti: rispetto alla composizione socio-profes-

${ }^{3}$ Sulle cause della fine dei regimi comunisti e sulle differenti modalità di dissoluzione del potere comunista («erosione» e «crollo») cfr. von Beyme (1992). 
sionale della classe politica comunista il cambiamento non poteva essere più radicale. Questa caratteristica, comune a tutti i movimenti di opposizione sorti in Europa orientale e nell'URSS nel 1989-1990, ha fatto parlare di «rivoluzione dell'intelligentsia» come fattore caratterizzante la caduta del comunismo (Cappelli 1990). La fine del sistema a partito unico e l'elezione del primo parlamento post-comunista sulla base di una competizione politica tra più partiti, hanno concluso la prima fase del processo di transizione dal regime comunista, segnata, in primo luogo, dall'adozione di un «nuovo» modello di rappresentanza: quella politica elettiva, «borghese».

Lo svolgimento delle elezioni politiche del giugno 1990 è stato preceduto dall'approvazione, da parte del parlamento provvisorio post-comunista, della legge sulla registrazione dei partiti politici, avvenuta il 23 gennaio 1990, in base alla quale ogni partito o movimento politico, al fine di potere partecipare alla competizione elettorale, è tenuto a formalizzare la propria esistenza presso il Ministero degli Interni, o presentando una petizione firmata da 10 mila sostenitori oppure dimostrando di godere del sostegno di almeno 10 mila elettori. Il 27 febbraio del 1990 è stata approvata la nuova legge elettorale che ha sancito l'adozione del sistema proporzionale di lista. Tale adozione è stata favorevolmente accolta sia dal Forum Civico che dal partito comunista e dagli altri partiti satellite, perché in grado di riflettere con cura tutte le opinioni politiche emerse dopo quaranta anni di forzato sostegno al regime comunista. In verità, il sistema proporzionale era stato già sperimentato dai cecoslovacchi della vecchia generazione: esso infatti era stato utilizzato sia nel periodo pre-bellico che nel 1946 (Wightman 1991). Il sistema adottato dalla legge elettorale del 1990 non ha preso, però, la forma del sistema proporzionale «puro», dato che la principale preoccupazione dei legislatori è stata quella di impedire la frammentazione partitica che aveva caratterizzato i parlamenti cecoslovacchi prima della seconda guerra mondiale. A tal fine venivano frapposti due ostacoli alla proliferazione dei partiti: la dimostrazione, da parte di ogni organizzazione partitica, di avere almeno 10 mila membri o di essere sostenuta da altrettanti elettori e l'introduzione di una soglia di esclusione, in base alla quale ogni partito o coalizione doveva ottenere almeno il 5\% dei voti all'Assemblea Federale e al Consiglio Nazionale Ceco e il 3\% al Consiglio Nazionale Slovacco (Pehe 1990, n. 22). Ancora, rispetto al vecchio sistema proporzionale di lista 
adottato nel periodo pre-comunista, il nuovo intendeva limitare l'eccessivo potere della macchina partitica e attribuire una maggiore influenza alle singole personalità; gli elettori potevano infatti esprimere fino a un massimo di quattro voti di preferenza.

La legge elettorale adottata per le elezioni del 1992, approvata dal parlamento federale il 29 gennaio dello stesso anno, è apparsa piuttosto simile a quella precedente nelle sue caratteristiche di fondo, anche se ha presentato delle novità di non poco rilievo. Il sistema elettorale adottato, contrariamente alla proposta fatta da Havel, è risultato essere ancora quello proporzionale di lista ${ }^{4}$. Anche la nuova legge elettorale ha previsto la suddivisione del territorio della federazione in 12 distretti elettorali corrispondenti alle dieci regioni del paese e alle due capitali, Praga e Bratislava; ogni partito ha continuato ad avere la facoltà di presentare due liste in ciascun distretto, una, cioè, per ciascuna camera. È stata data la possibilità ai partiti rappresentati nel parlamento del 1990 di essere automaticamente registrati, mentre gli altri hanno dovuto seguire le procedure per la registrazione già previste nella legge elettorale del 1990 (BBC SWB, 1 febbraio 1992) $)^{5}$. E stata data, inoltre, la possibilità all'elettore di modificare l'ordine di lista, così come previsto dalla legge elettorale del 1990 (Pehe 1992, n. 7). La nuova legge elettorale si è differenziata invece dalla precedente in quanto ha abbassato dal $50 \%$ al $30 \%$ il quorum per l'elezione dei candidati scelti al di fuori dell'ordine di lista. Allo scopo di contenere la prevedibile frammentazione partitica anche la nuova legge ha mantenuto immutata la soglia elettorale del $5 \%$, ma ha previsto soglie diverse per le coalizioni: il $7 \%$ per quelle costituite $\mathrm{da}$ due o tre partiti; il $10 \%$ per le coalizioni formate da più di quattro partiti. Nel caso in cui nessun partito o coalizione fosse stato in grado di superare tali soglie, queste ultime sarebbero state ridotte rispettivamente a $3 \%, 5 \%$ e $8 \%$. Le disposizioni legislative contenute nella legge elettorale federale sono state recepite anche dai parlamenti repubblicani (BBC - SWB, 29 feb-

${ }^{4}$ L'approvazione della nuova legge elettorale era stata preceduta dalla presentazione, da parte del presidente Havel, di una proposta di riforma della legge elettorale in senso uninominale. L'obiettivo di tale progetto era quello di dare maggiore voce alle singole personalità e di limitare la frammentazione partitica. Cfr. Radio Czechoslovakia 1700 gmt, 16 gennaio 1992, in «BBC - Summary of World Broadcasts», 22 gennaio 1992.

5 BBC - SWB è la sigla abbreviata del bollettino quotidiano della «BBC - Summary of World Broadcasts»; d'ora in poi: BBC - SWB. 
braio 1992): La nuova legge elettorale ha stabilito, inoltre, l'obbligo per tutti i partiti di comunicare, almeno tre giorni prima della chiusura della campagna elettorale, lo screening dei candidati per verificare se essi avessero o meno collaborato con la ex polizia segreta cecoslovacca (CSTK - 1992 Elections in Czechoslovakia, n. 1$)^{6}$.

L'adozione della legge elettorale proporzionale del 1992 è stata preceduta da un acceso dibattito politico attorno alla opportunità di mantenere il sistema proporzionale, o piuttosto di introdurre il maggioritario. Tra i fautori del sistema maggioritario, Havel, ad esempio, ha messo in correlazione la elevata frammentazione partitica e relativa instabilità governativa, esistente in Cecoslovacchia durante il periodo tra le due guerre, con la crescente differenziazione dello spettro partitico manifestatasi a partire dalla seconda metà del 1990. Per difendersi dalla «dittatura dei partiti» l'ex presidente cecoslovacco ha sostenuto l'opportunità di dare la possibilità agli elettori di scegliere le singole persone al di fuori di logiche di appartenenza partitica $\mathrm{e}$ in modo tale che $\mathrm{i}$ deputati eletti risultino maggiormente responsabili nei confronti del proprio specifico elettorato piuttosto che verso le rispettive organizzazioni di partito (Havel 1992).

La questione degli effetti dei sistemi elettorali sul sistema partitico è stata da sempre al centro del dibattito politologico occidentale. Le domande a questo proposito vertono sulla efficacia dei vari tipi di sistemi elettorali e sulla loro capacità di rivelarsi più adatti a seconda delle varie caratteristiche dei sistemi sociali. Ha il sistema proporzionale puro un effetto moltiplicativo sul numero dei partiti, come ha sostenuto Duverger (1961), o si limita invece a fotografare la realtà politica organizzata, lasciando «ai partiti la libertà di moltiplicarsi al negativo, semplicemente perché non ne penalizza la moltiplicazione» (Sartori $1990,251)$ ? E sufficiente ridurre il grado di proporzionalità del sistema elettorale, tramite ad esempio soglie di esclusione, in modo da limitare la frammentazione del sistema partitico? E ancora, è opportuno fare scelte ponderate di ingegneria costituzionale in paesi, come la Cecoslovacchia, che intendono instaurare un regime democratico? In realtà alla situazione socio-politica cecoslovacca, di per sé poco coesa ed omogenea, non sem-

${ }^{6}$ CSTK sta per: Ceskoslovensky Tiskova Kancelar (agenzia stampa cecoslovacca). 
brerebbe confacente un sistema maggioritario uninominale, quanto, invece, un sistema proporzionale, sia pure corretto, se è vero che «in una società ove sono presenti diverse minoranze etniche e culturali, geograficamente concentrate, un sistema bipartitico può non solo non essere auspicabile, ma anche completamente impossibile; il sistema elettorale a scrutinio maggioritario uninominale non è sicuramente un mezzo sufficiente a realizzarlo» (Lijphart 1984, 241).

\section{Il sistema partitico alla prova elettorale del 1990}

Così come si è verificato nel corso dei processi di transizione dall'autoritarismo alla democrazia nei paesi dell'Europa meridionale (Italia, Spagna, Portogallo, Grecia), anche in Cecoslovacchia i partiti politici hanno ricoperto il ruolo di attori principali della transizione. Infatti, pure nella realtà dei paesi dell'Europa orientale il passaggio dal regime comunista al sistema democratico è stato contrassegnato dalla presenza di vari partiti politici, espressione di un pluralismo in grado di allontanare maggiormente i pericoli di svolte autoritarie (Morlino 1986a; 1992). Le diffidenze espresse dall'elettorato cecoslovacco nei riguardi dell'istituzione partitica (Rak 1990), retaggio del recente passato comunista, non hanno effettivamente impedito un vero e proprio exploit di partecipazione politica: tra la fine di novembre del 1989 e la fine di gennaio del 1990 sono nati, infatti, in Cecoslovacchia circa 40 gruppi e movimenti politici (Pehe 1990, n. 10), e alla fine del luglio 1990 ne sono stati registrati circa 100. Il sistema proporzionale corretto ha impedito, però, che i parlamenti, federale, ceco e slovacco, eletti nel giugno del 1990 e nel giugno del 1992, fossero caratterizzati da una elevata frammentazione partitica: solo relativamente poche formazioni politiche, rispetto alle numerose che hanno partecipato alle elezioni, sono riuscite a superare i vari sbarramenti elettorali (cfr. tabb. 1 e 2 ).

I risultati elettorali del giugno 1990 hanno palesato le peculiarità delle prime elezioni politiche del dopo comunismo. La schiacciante vittoria del Forum Civico nella Repubblica ceca (49,49\% dei voti) e del movimento Cittadini contro la Violenza in quella slovacca $(29,34 \%$ dei suffragi) ha dimostrato come in queste elezioni l'elettore non abbia espresso un voto di sostegno a un determinato partito dello spettro politico, ma un voto 
TAB. 1. Risultati delle elezioni per la Camera del popolo nel 1990 e nel 1992

Elezioni 1990

\begin{tabular}{lcc}
\hline Partiti & Voti $\%$ & Seggi \\
Forum Civico/Cittadini contro la Violenza & 46,6 & 87 \\
Partito Comunista & 13,6 & 23 \\
Unione Democratico-Cristiana & 12 & 20 \\
Associazione per la Moravia e la Slesia & 5,4 & 9 \\
Partito Nazionale Slovacco & 3,5 & 6 \\
Coesistenza & 2,8 & 5 \\
Totale & & 150 \\
\hline
\end{tabular}

Fonte: Keesing's Record of World Events, News Digest, giugno 1990.

Elezioni 1992

Partiti

Partito Civico Democratico/Partito Democratico-Cristiano

*Movimento per la Slovacchia Democratica

Blocco di Sinistra

*Partito della Sinistra Democratica

Partito Socialdemocratico Cecoslovacco

Partito Repubblicano Cecoslovacco

Unione Democratico Cristiana/Partito del Popolo Cecoslovacco

Unione Liberale Sociale

*Partito Nazionale Slovacco

* Movimento Cristiano Democratico

${ }^{\star}$ Coalizione dei Partiti Ungheresi

Totale

* Si tratta di partiti delle terre slovacche.

Fonte: CSTK in English 1331 gmt, 10 giugno 1992, in BBC-SWB, giugno 1992.

plebiscitario a favore o contro il passato regime: la scelta elettorale infatti ha assunto nettamente la forma della opzione tra comunismo ed anticomunismo. Con l'abolizione del principio del monopolio del potere del partito comunista e l'introduzione del multipartitismo i comunisti, pur avendo ottenuto oltre il 13\% dei suffragi, hanno perso la posizione privilegiata di cui godevano all'interno del sistema. Il Partito Comunista «riformato», nonostante la proclamata adesione ai nuovi valori liberal-democratici e l'espulsione dal partito di funzionari corrotti e dei personaggi politici più compromessi col precedente regime, non è riuscito a proporsi come valido partner politico né di governo né di opposizione. 
TAB. 2. Risultati delle elezioni per la Camera delle Nazioni nel 1990 e nel 1992

Elezioni 1990

\begin{tabular}{lcc}
\hline Partiti & Voti $\%$ & Seggi \\
Forum Civico/Cittadini contro la Violenza & 45,9 & 83 \\
Partito Comunista & 13,7 & 24 \\
Unione Democratico-Cristiana & 11,3 & 20 \\
Associazione per la Moravia e la Slesia & 3,6 & 9 \\
Partito Nazionale Slovacco & 6,2 & 7 \\
Coesistenza & 2,7 & 7 \\
Totale & & 150 \\
\hline
\end{tabular}

Fonte: Keesing's Record of World Events, New Digest, giugno 1990.

Elezioni 1992

\begin{tabular}{lcc}
\hline Partiti & Voti \% & Seggi \\
Partito Civico Democratico/Partito Democratico-Cristiano & 33,43 & 37 \\
*Movimento per la Slovacchia Democratica & 33,85 & 33 \\
Blocco di Sinistra & 14,48 & 15 \\
^Partito della Sinistra Democratica & 14,04 & 13 \\
Partito Socialdemocratico Cecoslovacco & 6,8 & 6 \\
Partito Repubblicano Cecoslovacco & 6,37 & 6 \\
Unione Democratico Cristiana/Partito del Popolo Cecoslovacco & 6,08 & 6 \\
Unione Liberale Sociale & 6,06 & 5 \\
${ }^{\star}$ Partito Nazionale Slovacco & 9,35 & 9 \\
*Movimento Cristiano Democratico & 8,81 & 8 \\
*Coalizione dei Partiti Ungheresi & 7,39 & 7 \\
${ }^{*}$ Partito Social-Democratico in Slovacchia & 6,09 & 5 \\
& & \\
Totale & & 150 \\
\hline
\end{tabular}

* Si tratta di partiti delle terre slovacche.

Fonte: CSTK in English 1331 gmt, 10 giugno 1992, in BBC-SWB, giugno 1992.

L'ottima performance elettorale dei due principali movimenti di opposizione (Forum Civico e Cittadini contro la Violenza), se ha sancito la fine del monopolio del potere comunista e la vittoria di leadership alternative, ha mostrato, allo stesso tempo, uno spettro politico scarsamente differenziato. Molti dei nuovi partiti e delle formazioni politiche «rinnovate» non sono riusciti a superare la soglia del $5 \%$. I loro programmi, in realtà, sono apparsi piuttosto generici, senza capacità effettive di articolare ed aggregare interessi di gruppi e strati sociali ben definiti. Dei gruppi politici di nuova formazione solo l'Associazione per la Moravia e la Slesia, con il $10,30 \%$ dei voti, è riuscita a 
superare lo sbarramento elettorale ed ottenere rappresentanza parlamentare, e questo grazie ad una campagna elettorale centrata sulla autonomia regionale in contrapposizione al sistema centralista. E ancora, l'accoglimento nel programma politico del Forum Civico di opzioni ecologiste ha costituito uno dei motivi principali dell'insuccesso di un movimento single-issue come $\mathrm{i}$ Verdi, i quali sono, invece, riusciti ad accedere al parlamento slovacco con il 3,44\% dei suffragi. La sconfitta elettorale del Partito Socialista e di quello Social-Democratico, due dei più importanti partiti storici, è invece dipesa soprattutto dalla loro, più o meno forzata, collaborazione col passato regime e dall'esigenza, fortemente sentita dall'elettorato cecoslovacco, di esprimere un voto di rottura, chiaramente anti-autoritario.

L'adozione di una soglia elettorale più bassa $(3 \%)$ per il Consiglio Nazionale slovacco ha determinato l'affermazione di un numero maggiore di partiti rispetto al parlamento ceco. Il movimento Cittadini contro la Violenza è uscito dalla consultazione elettorale del 1990 come la forza principale del panorama politico slovacco (29,3\% dei voti), seguito dal Movimento Democratico Cristiano (19,21\%). I comunisti slovacchi, che hanno prodotto una profonda revisione della loro immagine e introdotto numerosi cambiamenti nel programma e nell'organizzazione del partito (Weiss 13 giugno 1991), pur non avendo raggiunto una affermazione elettorale superiore a quella del partito comunista ceco, sono riusciti a conseguire un minore isolamento nell'ambito dell'opinione pubblica rispetto al partito comunista di Boemia e Moravia.

A partire dalle elezioni del 1990 è diventato sempre più evidente che l'obiettivo principale della Repubblica slovacca non consisteva più nel proposito di liberarsi dal dominio comunista, ma, soprattutto, in quello di impostare su nuove basi i rapporti politici ed economici tra le due Repubbliche della federazione. La competizione elettorale del giugno 1990 ha infatti concluso una tappa, a partire dalla quale è iniziata una nuova fase in cui le rivendicazioni nazionali slovacche hanno conosciuto una vera e propria impennata, non essendo più ostacolate dalle incognite connesse all'esito della rivoluzione anti-comunista.

\section{La fluidità del sistema: il caso del Forum Civico}

Una situazione di estrema fluidità del sistema partitico ha caratterizzato i due anni intercorsi tra le elezioni politiche del 
1990 e quelle del 1992. A partire dalla seconda metà del 1990 il «sistema partitico» cecoslovacco ha subito, infatti, una serie di modifiche che ne hanno trasformato completamente la conformazione. Nuove formazioni politiche sono nate; altri soggetti politici hanno costituito delle coalizioni tra partiti ideologicamente omogenei. I grandi movimenti politici che hanno guidato la «rivoluzione di velluto» del $1989-90$ si sono sciolti e divisi in più partiti. L'evoluzione subita da questi ampi movimenti di aggregazione di cittadini e partiti politici ha mostrato che Forum Civico e Cittadini contro la Violenza sono stati dei fenomeni di transizione, anche se molto importanti. Essi hanno, prima di tutto, assicurato un mutamento pacifico dal regime comunista al sistema democratico; in secondo luogo, hanno offerto una leadership alternativa e coesa al momento del crollo del regime; inoltre, essi hanno costituito un luogo di mobilitazione e di azione collettiva di grande rilevanza. L'esito positivo della rivoluzione e l'apparente irreversibilità dei cambiamenti fino a quel momento realizzati, hanno fatto venir meno la necessità di tenere aggregato quel blocco di forze riunite sotto la bandiera del Forum Civico e di Cittadini contro la Violenza.

Il Forum Civico, nato il 19 novembre del 1989, l'attore politico più importante durante la prima fase di transizione dal regime comunista, ha conosciuto nel corso della propria esistenza mutamenti profondi. Uno dei suoi primi atti politici è stato l'avvio di negoziati con la leadership comunista cecoslovacca e la richiesta di dimissioni di tutti gli esponenti politici responsabili degli eventi del '68 (Pehe 19 dicembre 1989). Esso si è presentato come un'ampia coalizione centrista di partiti politici, gruppi civici ed intellettuali di orientamento liberale (Rak 1990). I principi cardine del suo programma politico sono stati incentrati sulla richiesta di una nuova costituzione e di nuove istituzioni democratiche, di libere elezioni, della fine del monopolio del partito comunista, del rispetto dei diritti sociali e delle nazionalità, nella difesa del mercato e dell'uguaglianza tra i vari tipi di proprietà. Tuttavia, la mancanza di una forte organizzazione e disciplina di partito e di una chiara identità politica, la pluralità di vedute, che sono stati motivi di successo nel corso della prima metà del 1990 , sono diventati in seguito le cause principali della crisi del Forum Civico (Pehe 1990, n. 25). Il processo di disgregazione di questo movimento è stato anche l'esito finale di un processo di differenziazione interna, cominciato a partire dalla seconda metà del 1990 , sulle principali que- 
stioni socio-economiche. L'applicazione delle prime misure di politica economica ha infatti acuito le discussioni e i contrasti tra le varie correnti del Forum Civico. La politica radicalmente liberista intrapresa dall'allora Ministro delle Finanze Vaclav Klaus è divenuta oggetto principale delle polemiche, specialmente da parte dei gruppi di sinistra del Forum; sono state, soprattutto, le modalità, i tempi della privatizzazione e la scarsa considerazione di misure sociali atte a difendere i ceti più colpiti dalla transizione ad essere maggiormente criticati. Il processo di trasformazione è stato caratterizzato da un lungo dibattito congressuale durato più di sei mesi, a conclusione del quale le opposte fazioni hanno dato vita a distinti gruppi parlamentari (Pehe e Obrman 1990, n. 47): il Club Interparlamentare della Destra Democratica, dal quale è nato, al congresso del Forum Civico del gennaio 1991, il Partito Democratico Civico, presieduto da Vaclav Klaus'; il Club Liberale del Forum Civico, che ha assunto poi il nome di Movimento Civico, ed il partito di destra Alleanza Democratica Civica (Pehe 1991, n. 10; Pehe 1991, n. 3). Alcuni dei gruppi di sinistra presenti nel Forum, quali il Club Rinascita, o deputati come Ivan Fisera, Valtr Komarek, Milos Zeman, Zdenek Jicinsky e Petr Kucera, sono, invece, confluiti nel Partito Social-Democratico Cecoslovacco (Pehe 1990, n. 43; Hajek 9 giugno 1992).

Le elezioni politiche del 1992 e l'acuirsi della polarizzazione politica

I profondi mutamenti politici, intervenuti in Cecoslovacchia nel corso dei due anni tra la prima e la seconda consultazione

7 V. Klaus, il principale artefice della riforma economica cecoslovacca e sostenitore di una privatizzazione veloce, è nato nel $1941 \mathrm{e}$ ha conseguito la laurea alla Scuola di Economia di Praga nel 1963; nel 1966 ha studiato in Italia e nel 1969 negli USA. Tra il '63 e il '70 ha lavorato all'Istituto Economico dell'Accademia delle Scienze Cecoslovacca. Dopo il suo licenziamento dall'Istituto Economico, a causa delle sue idee antimarxiste, è stato assunto dalla Banca di Stato Cecoslovacca e nel 1988-89 dall'Istituto di Previsioni dell'Accademia delle Scienze Cecoslovacca. È stato membro del Centro di Coordinamento del Forum Civico a partire dal novembre del 1989 e nel dicembre del 1989 gli è stato affidato l'incarico di Ministro delle Finanze, carica alla quale è stato riconfermato in seguito alle elezioni del 1990. Attualmente ricopre la carica di Primo Ministro della Repubblica ceca. Cfr. BBC - SWB, 22 ottobre 1990. Sull'elezione di Klaus come presidente del partito cfr. Keesing's Record of World Events - News Digest for October 1990, p. 37791. 
politica dell'era post-comunista, che hanno poi condotto all'inizio del 1993 allo scioglimento della Federazione e alla costituzione di due Stati indipendenti, hanno fatto emergere una situazione di elevato pluralismo partitico. Oltre quaranta formazioni politiche hanno infatti concorso alle elezioni del giugno 1992; di queste, però, solo dodici hanno superato gli sbarramenti elettorali ed ottenuto seggi nel Parlamento Federale, e solo otto nel Consiglio Nazionale ceco. Il parlamento slovacco appare, rispetto a quello ceco, caratterizzato da un «multipartitismo moderato» (Sartori 1976), in quanto costituito da cinque partiti.

La frattura politica creatasi tra le due Repubbliche ha costituito una delle novità più significative emerse nel corso delle ultime elezioni politiche cecoslovacche. Motivazioni e obiettivi diversi hanno mosso gli elettorati ceco e slovacco.

Nella Repubblica ceca i partiti di centro-destra, e in particolare il Partito Democratico Civico, l'Alleanza Democratica Civica e il Partito del Popolo, hanno ottenuto oltre il $40 \%$ dei suffragi elettorali. Il quasi $30 \%$ dei voti ottenuto dal Partito Democratico Civico nel Consiglio Nazionale ceco mostra il consenso, piuttosto elevato, di cui gode il suo programma liberista e in particolare la «terapia shock» di Klaus.

La sconfitta del centro politico, cioè del Movimento Civico e, assieme ad esso, della maggior parte della classe politica di governo del periodo post-comunista, appartenente per lo più al disciolto movimento Cittadini contro la Violenza e al Movimento Civico, ha rappresentato una delle sorprese di queste elezioni (Obrman 1992, n. 26).

La sinistra ceca, costituita dal Partito Comunista di Boemia e Moravia, dal Partito Social-Democratico e dall'Unione Liberale Sociale, non ha visto crescere di molto i suoi consensi: ha infatti ottenuto poco più del $27 \%$ di voti.

Un gruppo regionalista, l'Associazione per la Moravia e la Slesia, già presente nel parlamento eletto nel 1990, ha superato la soglia del $5 \%$, continuando a mantenere la questione dell'autonomia di queste due regioni all'ordine del giorno dell'agenda politica. Rispetto alle precedenti elezioni ha, però, ottenuto un numero minore di consensi (5,87\% di suffragi) (cfr. tab. 3 ).

A seguito delle elezioni del 1992, forti differenze sono state riscontrate in Slovacchia, sia rispetto alle formazioni politiche prevalse nella Repubblica ceca, sia rispetto alla composizione stessa del parlamento slovacco eletto nel 1990. Qui un forte 
TAB. 3. Risultati delle elezioni per il Consiglio Nazionale ceco nel 1990 e nel 1992

Elezioni 1990

\begin{tabular}{lcc}
\hline Partiti & Voti $\%$ & Seggi \\
Forum Civico & 49,49 & 127 \\
Partito Comunista & 13,23 & 32 \\
Associazione per la Moravia e la Slesia & 10,3 & 22 \\
Unione Democratico Cristiana & 8,41 & 19 \\
Totale & & 200 \\
\hline
\end{tabular}

Fonte: Obrman (1990), Civic Forum Surges to Impressive Victory in Elections, in «RFE», I, n. 25.

Elezioni 1992

Partiti

Partito Civico Democratico

Blocco di Sinistra

Partito Social-Democratico Cecoslovacco

Unione Liberale Sociale

Unione Democratico Cristiana-Partito del Popolo Cecoslovacco

Associazione per la Moravia e la Slesia

Alleanza Democratica Civica

Partito Repubblicano Cecoslovacco

\section{Voti $\% \quad$ Seggi}

$29,73 \quad 76$

$14,05 \quad 35$

$6,53 \quad 16$

$6,52 \quad 16$

$6,28 \quad 15$

$5,87 \quad 14$

$5,93 \quad 14$

$5,98 \quad 14$

200

Totale

Fonte: (1992) Slovak Radio, Bratislava $1630 \mathrm{gmt}, 7$ giugno 1992, in BBC - SWB, 9 giugno; (1992) Radio Czechoslovakia 2000 gmt, 9 giugno 1992, in BBC - SWB, 11 giugno; Pehe, J. (1992), Czechoslovakia's Political Balance Sheet, 1990 to 1992, in «RFE/ RL», I, n. 25.

movimento nazionalista, il Movimento per una Slovacchia Democratica, guidato da Vladimir Meciar, ha ottenuto la maggioranza dei suffragi elettorali $(37,26 \%)$, diventando così il primo partito slovacco.

Il Movimento Democratico Cristiano dell'ex Primo Ministro slovacco Carnogursky ha visto una forte diminuzione dei propri consensi elettorali, principalmente a causa dei suoi orientamenti pro-federali: esso ha infatti registrato una perdita di oltre il $50 \%$ dei consensi, passando dal $19,20 \%$ all' $8,9 \%$ dei voti.

In Slovacchia i partiti di centro-destra e, in generale, quelli favorevoli al mantenimento della federazione cecoslovacca, come ad esempio l'Unione Democratica Civica (ex $\mathrm{CcV}$ ) o la coalizione formata dal Partito Democratico Civico e dal Partito Democrati- 
TAB. 4. Risultati delle elezioni per il Consiglio Nazionale slovacco nel 1990 e nel 1992

Elezioni 1990

\begin{tabular}{lcc}
\hline Partiti & Voti \% & Seggi \\
Cittadini contro la Violenza & 29,34 & 48 \\
Movimento Democratico Cristiano & 19,2 & 31 \\
Partito Nazionale Slovacco & 13,94 & 22 \\
Partito Comunista & 13,34 & 22 \\
Coesistenza & 8,64 & 14 \\
Partito Democratico & 4,39 & 7 \\
Partito Verde & 3,4 & 6 \\
Totale & & 150 \\
\hline
\end{tabular}

Fonte: Obrman (1990), Civic Forum Surges to Impressive Victory in Elections, in «RFE», I, n. 25.

Elezioni 1992

Partiti

Voti $\% \quad$ Seggi

Movimento per la Slovacchia Democratica $37,26 \quad 74$

Partito della Sinistra Democratica

$14,7 \quad 29$

Movimento Democratico Cristiano

$8,88 \quad 18$

Partito Nazionale Slovacco

$7,39 \quad 15$

Coalizione Partiti Ungheresi

7,42

Totale

Fonte: (1992) Slovak Radio, Bratislava 1630 gmt, 7 giugno 1992, in BBC - SWB, 9 giugno; (1992) Radio Czechoslovakia 2000 gmt, 9 giugno 1992, in BBC - SWB, 11 giugno; Pehe, J. (1992), Czechoslovakia's Political Balance Sheet, 1990 to 1992, in «RFE/ RL», I, n. 25.

co, sono stati fortemente penalizzati dall'elettorato, raggiungendo rispettivamente appena il 4,0\% e il 3,3\% dei voti.

Il Partito della Sinistra Democratica (gli ex comunisti slovacchi) con oltre il $14 \%$ dei voti è diventato il secondo partito nel Consiglio Nazionale slovacco, anche se, rispetto alle elezioni del 1990, non ha registrato un consistente aumento dei suoi consensi.

Oltre al Movimento per una Slovacchia Democratica, un altro partito nazionalista, il Partito Nazionale Slovacco, ha ottenuto rappresentanza parlamentare; esso, pur registrando una perdita di circa il $6 \%$ di voti rispetto alla percentuale del 1990 $(13,94 \%)$, ha abbracciato un orientamento indipendentista molto più radicale che in passato (cfr. tab. 4). 
Il quadro politico-partitico delineatosi nelle due repubbliche all'indomani delle elezioni del 5 e 6 giugno 1992 si presenta fortemente polarizzato: da un lato la netta affermazione di partiti di centro-destra favorevoli alla continuazione delle riforme economiche in senso liberista; dall'altro il successo di gruppi nazionalisti e di centro-sinistra, fautori dell'instaurazione di un diverso rapporto politico-economico tra le due Repubbliche e di un processo di privatizzazione più lento e graduale. Rispetto alle elezioni del 1990, però, i maggiori spostamenti nelle preferenze elettorali si sono avuti in Slovacchia. Nella Repubblica ceca gli spostamenti si sono verificati all'interno della stessa area di centro-destra: se si sommano infatti i voti ottenuti dal Movimento Civico, dall'Alleanza Democratica Civica e dal Partito Democratico Civico, cioè dai partiti nati dallo scioglimento del Forum Civico, i risultati sono simili alla percentuale dei suffragi ottenuta allora dal Forum Civico (49,5\% dei voti) (RFE/ RL, n. 26, 1992).

La polarizzazione tra le due repubbliche ha avuto uno specifico riflesso sul livello di stabilità ed efficienza dell'ultimo parlamento federale.

L'Assemblea Federale era costituita dalla Camera del Popolo e dalla Camera delle Nazioni; quest'ultima era ulteriormente divisa in due: una composta da deputati cechi e l'altra da deputati slovacchi. Ogni legge per essere approvata necessitava della maggioranza nella Camera del Popolo e della maggioranza dei cechi e degli slovacchi separatamente. Una maggioranza di tre quinti era, invece, richiesta per ogni legge costituzionale e per l'elezione del Presidente della Repubblica (Obrman 1992, n. 41). La composizione partitica di ognuna delle due camere dell'Assemblea Federale non ha potuto che rispecchiare la frattura politica esistente nel paese. Così, la Camera del Popolo ha registrato la presenza di una maggioranza di centro-destra e pro-federale, composta dal Partito Democratico Civico, Partito del Popolo, Movimento Democratico Cristiano e dalla Coalizione di partiti Ungheresi; i partiti di sinistra (Blocco di Sinistra, Partito Social-Democratico, Unione Liberale Sociale e il Partito della Sinistra Democratica) hanno ottenuto 46 seggi, i partiti nazionalisti slovacchi 30 seggi e il Partito Repubblicano di estrema destra 8 seggi (BBC - SWB, 12 giugno 1992). Essendo i partiti di sinistra, quelli nazionalisti e il Partito Repubblicano accomunati da un medesimo obiettivo, quello, cioè, di modificare i tempi e le modalità della riforma economica, è apparsa 
alquanto remota la possibilità di cooperazione tra questi partiti e quelli di centro-destra (BBC - SWB, 10 giugno 1992). Nella Camera delle Nazioni si è assistito a una simile polarizzazione, con una Camera ceca dominata dai partiti liberisti e una Camera slovacca in cui i partiti nazionalisti e di sinistra hanno il predominio. In tali condizioni il Parlamento Federale ha assunto sempre più le sembianze di un organo di transizione, dominato sostanzialmente da due partiti (il Partito Democratico Civico e il Movimento per una Slovacchia Democratica), e incapace di legiferare sulle questioni politiche cruciali $(\mathrm{BBC}-\mathrm{SWB}, 3$ ottobre 1992).

\section{Le questioni aperte dalla transizione}

Il reclutamento del personale politico tramite libere elezioni competitive tra più partiti ha costituito per i riformatori postcomunisti, sia in Cecoslovacchia che negli altri ex paesi comunisti dell'Europa Orientale, l'obiettivo primario della loro attività politica e, al tempo stesso, lo strumento fondamentale per la costruzione di uno stato democratico. Le elezioni del 5 e 6 giugno 1992, assieme a quelle del giugno 1990, hanno rappresentato, infatti, tappe importanti del processo di transizione della Cecoslovacchia dal regime comunista.

L'analisi di queste due tornate elettorali pone la questione se possono i nuovi partiti e movimenti politici nati dopo il crollo del regime comunista considerarsi espressione di diversi interessi di classe, se vanno, cioè, disponendosi lungo la frattura socio-economica tipica degli allineamenti elettorali presenti in molte società industriali europee (Lipset e Rokkan 1967). La risposta deve essere cauta. Innanzitutto, in molti casi, i programmi e gli appelli presentati durante la campagna elettorale sembrano riflettere la fluidità della fase di transizione che il paese sta ancora vivendo e il non ancora avvenuto consolidamento del sistema partitico (Morlino 1986b). Ciò che emerge in maniera particolare dalla competizione tra i vari partiti, operanti all'interno dello spettro rappresentativo cecoslovacco, è la centralità nel dibattito politico di due cleavages intersecantisi. Da un lato la issue della privatizzazione economica, sui tempi e sull'ampiezza della quale si è creata tra le diverse forze politiche una prima frattura che potrebbe essere ricondotta, in senso lato, ad una frattura di classe. Dall'altro, la questione dell'auto- 
nomia della Repubblica slovacca dalla federazione dominata dai cechi, che ha assunto la configurazione di un cleavage nazionale, cioè, di una frattura tra centro e periferia che è stata all'origine della «rinascita» dei partiti nazionalisti slovacchi ${ }^{8}$ e che ha contribuito a rendere più difficile il processo di transizione $\mathrm{da}$ un regime autoritario a un sistema democratico (Pridham 1991).

Il tema che ha dominato la campagna elettorale del 1990 è stato la sconfitta del comunismo e della posizione di monopolio del partito comunista (Martin 1990, n. 24); le elezioni del 1992 hanno, invece, ruotato attorno alla questione nazionale e a quella del proseguimento della riforma economica iniziata nel 1990. Inoltre, nei due anni trascorsi tra i due appuntamenti elettorali sono scomparsi i grandi movimenti che avevano guidato la «rivoluzione di velluto» e al loro posto sono nati nuovi partiti politici. Quasi tutti i leaders dei governi post-comunisti, soprattutto quelli del Movimento Civico', non sono stati rieletti e soltanto il $17 \%$ degli ex membri dell'Assemblea Federale ha visto riconfermato il proprio mandato elettorale il 5 e 6 giugno 1992 (Obrman 1992, n. 26). La mancata elaborazione di specifiche piattaforme programmatiche che potessero differenziare fra loro i vari gruppi politici, la sottovalutazione dell'importanza di una forte ed efficiente organizzazione partitica e di un diverso rapporto con l'elettorato, sono questi i principali fattori per comprendere come partiti quali il Movimento Civico o l'Unione Democratica Civica (ex $\mathrm{CcV}$ ) non siano riusciti a superare lo sbarramento elettorale del $5 \%$.

Alle elezioni del 1992 nessun partito è riuscito ad ottenere i medesimi risultati elettorali in ciascuna delle due repubbliche, con il risultato di accentuare la polarizzazione esistente tra le

$8 \mathrm{La}$ frattura nazionale, e in particolar modo il rivendicazionismo autonomista slovacco, ha costituito una costante nella storia della Cecoslovacchia. L'eterogeneità etnica e culturale delle popolazioni cecoslovacche ha accresciuto sin dal periodo della formazione dello stato unitario la già elevata frammentazione partitica: ben quindici formazioni politiche, ognuno rappresentante di un gruppo etnico diverso, avevano operato nel periodo precedente all'instaurazione del regime comunista (Riosa 1974).

9 Il Movimento Civico, presieduto dall'ex dissidente ed ex Ministro degli Esteri Jiri Dienstbier, è una delle formazioni politiche nate dallo scioglimento del FC. Favorevole al mantenimento di una struttura organizzativa ampia e movimentista, dopo le elezioni del 1992 ha avviato un processo di trasformazione in moderno partito di tipo liberale. Sostenitore di un profondo processo di trasformazione dell'economia cecoslovacca, ritiene, al tempo stesso, importante l'adozione di misure di welfare a difesa dei ceti sociali colpiti dalla transizione economica. 
due società. È emersa infatti, in tutta evidenza, la fragilità dell'assetto federale e la netta divaricazione, in termini di scelte partitiche e di priorità, esistente tra la Repubblica ceca e quella slovacca. Se nella prima, infatti, e in modo particolare a Praga, la maggior parte dell'elettorato ha votato per i partiti di centrodestra, nella Slovacchia hanno vinto i partiti nazionalisti e le sinistre (Obrman 1992, n. 26).

La riforma economica ha costituito uno dei principali motivi di disaccordo tra i partiti cechi e quelli slovacchi, facendo così emergere una ulteriore differenza rispetto alle elezioni del 1990. Se, allora, tutti i partiti della federazione, tranne i comunisti, avevano rifiutato in modo netto i principi dell'economia collettivista e sostenuto il programma di privatizzazione economica, adesso gli obiettivi, le modalità e i tempi della riforma economica sono stati sottoposti a critiche, soprattutto dai partiti della Repubblica slovacca. A differenza della maggior parte dei partiti cechi, ed in particolare di quelli di centro-destra, tutti i partiti slovacchi presenti in parlamento hanno sostenuto la necessità di una riforma economica graduale, capace di tenere conto delle specifiche condizioni socio-economiche della Slovacchia ${ }^{10}$, di misure sociali in difesa dei ceti deboli e di un maggiore potere di intervento dello stato in economia.

La questione nazionale e il problema delle prospettive future della federazione ceco-slovacca, manifestatisi sin dall'inizio della «rivoluzione di velluto» come conseguenza di un incompiuto sviluppo statuale della Slovacchia e del permanere di profondi squilibri economico-sociali tra le due repubbliche, hanno, però, dominato la campagna elettorale del 1992, trasformando le elezioni in una sorta di referendum pro o contro la conservazione dello Stato comune. Ogni elettore è stato, in pratica, chiamato a scegliere tra le varie soluzioni alla questione territoriale prospettate dai partiti. In realtà le divergenze tra cechi e slovacchi erano divenute subito manifeste sin dalla fine del 1989: se per i primi il modello politico cui fare riferimento era la Prima Repubblica fondata nel 1918 da Tomas Garrigue Masarik, per gli slovacchi la ricerca di una propria identità, espressasi anche

${ }^{10} \mathrm{La}$ Slovacchia, economicamente più debole e con uno sviluppo industriale di impronta sovietica, che ha privilegiato la diffusione delle industrie pesanti, si trova ad affrontare problemi, sia strutturali che sociali, ben più gravi in questa fase di transizione da un'economia pianificata a una di mercato. I tassi di disoccupazione sono, ad esempio, tre volte più alti di quelli della Repubblica ceca. 
con rivendicazioni riguardanti il nome della federazione e l'introduzione dello slovacco come lingua ufficiale ${ }^{11}$, nonché lo sviluppo di un rapporto paritario con la Repubblica ceca risultavano essere gli obiettivi prioritari ${ }^{12}$. Tale nazionalismo slovacco tuttora, però, non è riuscito a ripianare certe ambiguità nei confronti della democrazia. Così, il periodo che la Slovacchia ha vissuto tra il 1939 e il 1945, sotto il governo filonazista di Monsignor Tiso, ha visto confrontarsi due diverse tradizioni politiche: una, di segno nazional-conservatore-populista, che fa riferimento alla dichiarazione di indipendenza dello Stato Slovacco del 1939; la seconda, a carattere nazionalista-europeista, che si riconosce nella Resistenza allo stato slovacco fascista e tende verso una società più aperta e pluralista (Butora, Butorova e Rosova 1991). Il nazionalismo della Slovacchia, visto anche come possibilità di intraprendere una via autonoma e diversa di transizione all'economia di mercato, si è via via trasformato in una vera e propria frattura politica, sulla quale ogni partito dello spettro politico slovacco ha dovuto necessariamente confrontarsi. Quei partiti slovacchi che non hanno fatto chiare scelte autonomiste o che, comunque, non si sono pronunciati a favore di un ruolo ed un rapporto diversi della Slovacchia nei confronti dei cechi, sono stati penalizzati elettoralmente. Su tale issue la lacerazione con i partiti cechi non poteva essere più profonda; nella Repubblica ceca infatti hanno vinto i partiti sostenitori della federazione e contrari alle rivendicazioni nazionali-

$"$ Circa la disputa sul nome dello stato, cfr. Keesing's Record of World Events News Digest for March 1990 e News Digest for April 1990. Sul problema della lingua cfr. Keesing's Record of World Events - News Digest for October 1990.

12 La specificità della nazione slovacca rispetto a quella ceca nasce, oltre che da una elevata eterogeneità etnica, soprattutto da diverse e distinte caratteristiche culturali, economiche e politiche. Il problema dell'indipendenza slovacca ha radici molto antiche e le ostilità col centro dell'impero austro-ungarico risalgono al 1848, quando la Slovacchia, sottoposta al dominio magiaro, si ribellò non all'impero, ma al dominio ungherese.

Quando nel 1918 la Cecoslovacchia divenne uno stato unitario e centralizzato, le rivendicazioni slovacche si rivolsero contro il centro di Praga e contro Masarik, colpevoli di non aver mantenuto le promesse di autonomia fatte alla Slovacchia al momento dell'accordo di Pittsburgh nel 1918.

Nel 1938, durante l'occupazione nazista, la Slovacchia divenne uno stato indipendente fascista e antisemita guidato da Monsignor Tiso. Nel 1945, dopo la vittoria delle forze democratiche, la Cecoslovacchia non fece in tempo ad affrontare la questione slovacca, in quanto ben presto l'instaurazione del regime comunista soffocò ogni forma di autonomia politica. Nel 1968, durante la Primavera di Praga, la Cecoslovacchia divenne una repubblica federale, ma la «normalizzazione» comunista ne impedì il reale funzionamento. Cfr. Mitchell (1992, n. 31). 
ste della Slovacchia. Un discorso a parte va, bensì, fatto per i partiti di sinistra, che si sono espressi a favore della conservazione dello Stato comune tra cechi e slovacchi, e hanno giudicato incostituzionali gli accordi tra Klaus e Meciar, più tardi conclusisi con lo scioglimento della federazione ${ }^{13}$. Tali partiti (in particolare il Partito Social-Democratico e il Partito della Sinistra Democratica) auspicavano lo svolgimento di un referendum, come solo e legittimo strumento per decidere dell'eventuale dissoluzione dello Stato comune (BBC - SWB, 27 luglio 1992; BBC - SWB, 30 luglio 1992).

13 I colloqui sul futuro della federazione Cecoslovacca tra Klaus (difensore della federazione) e Meciar (sostenitore di un assetto confederale) hanno avuto inizio subito dopo le elezioni del 5 e 6 giugno 1992 e si sono conclusi, dopo cinque incontri, con l'accordo del 22 e 23 luglio dello stesso anno. I due leader hanno deciso di sottoporre all'Assemblea Federale una bozza di legge costituzionale sulle modalità di scioglimento della federazione e sulla divisione del patrimonio delle due repubbliche. La legge costituzionale, discussa dal parlamento federale nella seconda metà del 1992, alla seduta dell' 1 ottobre 1992 non ha ottenuto i $3 / 5$ dei voti necessari per essere approvata. Ad essa si sono opposti principalmente i partiti di sinistra. Il 25 novembre 1992 l'Assemblea Federale è riuscita infine ad approvare le legge sulla dissoluzione della Federazione cecoslovacca. La legge era stata precedentemente bocciata dai partiti di opposizione, $i$ quali insistevano sulla necessità di svolgere un referendum per decidere le sorti dello stato comune. Il $1^{\circ}$ gennaio 1993 la Cecoslovacchia, dopo settantaquattro anni di vita, si è divisa in due Stati indipendenti. Cfr. Mitchell (1992, n. 30). Per gli ulteriori sviluppi della vicenda cecoslovacca cfr. Pehe (1992, n. 45 e n. 48$)$. 
APPENDICE: I PROGRAMMI ELETTORALI DEI PARTITI POLITICI

\section{I partiti dello spettro politico ceco}

Il Partito Democratico Civico (PDC), forte di circa 20 mila membri attivi, soprattutto giovani professionisti di origine urbana, è il partito più forte nella Repubblica ceca; alle elezioni del 1992, in coalizione con il Partito Democratico Cristiano ha conquistato oltre il $33 \%$ dei voti all'Assemblea Federale e il $29,73 \%$ di voti al Consiglio Nazionale ceco. I maggiori consensi per il $P D C$ sono venuti dai centri urbani e dagli elettori con uno status socio-occupazionale più elevato (Obrman 1992, n. 26). L'iscrizione al partito è stata sottoposta a nuova regolamentazione: chi si iscrive ad esso non può far parte di altri partiti; sono, inoltre, vietate forme di iscrizione collettiva. Fautore di una riforma economica radicale e di un sistema politico incentrato sui partiti (Pehe 1992, n. 5), il PDC ha un programma politico-economico di destra, ispirato ai principi dell'individualismo (Pehe 1991, n. 5). $\grave{E}$ infatti un partito nettamente contrario a ogni forma di collettivismo, e fortemente liberista in economia; auspica che la Cecoslovacchia si avvii verso un'economia di mercato attraverso un processo di privatizzazione veloce (CSTK, 1992 Elections in Czechoslovakia, n. 4, 1992). Come, infatti, ha affermato Vaclav Klaus, presidente del partito e principale architetto della riforma economica cecoslovacca: «La privatizzazione è la principale priorità di tutte le economie che veramente si riformano. Un massiccio spostamento di diritti di proprietà dal governo in mani private è essenziale se queste economie devono diventare sistemi di mercato. La privatizzazione deve essere realizzata rapidamente; migliaia di imprese statali devono essere de-nazionalizzate in alcuni mesi o anni» (Klaus 1992, 27). Il PDC fa riferimento nel suo programma elettorale alle tradizioni ed agli ordinamenti democratici occidentali e difende il sistema elettorale proporzionale, che, secondo Klaus, costituisce una sicura difesa dell'istituzione partitica (BBC SWB, 11 gennaio 1992).

Sul problema dell'ordinamento territoriale, partito da posizioni pro-federaliste, dopo le elezioni del giugno 1992 , si è spostato su posizioni scissionistiche, che il 22 e il 23 luglio 1992 hanno portato all'accordo tra Klaus e Meciar sullo scioglimento della federazione (Mitchell 1992).

Il Partito Democratico Cristiano, fondato nel dicembre del 1989, ha concorso alle elezioni del 1992 in coalizione col Partito Democratico Civico. E un partito ad orientamento conservatore, che condivide nelle linee principali il programma di centro-destra del $P D C$; sostenitore anch'esso di riforme economiche radicali, da realizzare per mezzo di una privatizzazione veloce e della restituzione della proprietà ai 
loro legittimi proprietari (CSTK, 1992 Elections in Czechoslovakia, $\mathrm{n}$. 3, 1992).

Sulla questione nazionale, si dichiarava a favore di una federazione tripartita, nella quale ogni repubblica fosse dotata di una propria amministrazione (CSTK, n. 1, 1992).

L'Alleanza Democratica Civica ( $A C D$ ), nata nel dicembre del 1989, ha partecipato alle elezioni del 1990 sotto la bandiera del FC, dal quale si è staccata in seguito allo scioglimento del movimento stesso, nel dicembre del 1990. Alle ultime elezioni politiche è riuscita a superare la soglia elettorale solo al Consiglio Nazionale ceco, conquistando il 5,93\% dei voti (BBC - SWB, 11 giugno 1992; Pehe 1992, n. 25). Politicamente molto vicina al $P D C$ di Klaus (Pehe 1991, n. 21), l'Alleanza Democratica Civica è espressione della destra democratica e delle correnti conservatrici occidentali (CSTK, n. 1, 1992). In campo economico l'obiettivo prioritario del partito è la transizione da una economia pianificata ad una di mercato, da realizzare tramite una privatizzazione veloce e la restituzione delle terre ai legittimi proprietari (CSTK, n. 4, 1992). Sul problema della restituzione delle terre, il vicepresidente del partito, Daniel Kroupa, in una intervista ha affermato: «Noi consideriamo la restituzione uno dei punti chiave della nostra piattaforma. Essa è la base della privatizzazione e del ripristino del diritto di proprietà» (Kroupa 1992).

Sul problema nazionale l' $A D C$ considerava il referendum il mezzo più appropriato per decidere sul futuro della federazione (CSTK, n. 4, 1992).

L'Unione Democratico Cristiana - Partito del Popolo Cecoslovacco, fondata nel giugno del 1991 (CSTK, 1992 Elections in Czechoslovakia, n. 2, 1992), alle elezioni del 1992 ha ottenuto il $6,28 \%$ dei voti al parlamento ceco e il 5,98\% all'Assemblea Federale. Nel suo programma elettorale viene sottolineata l'importanza dei valori democratici e cristiani. Ė a favore dello sviluppo dell'economia di mercato e ritiene che lo stato debba limitare al minimo i propri poteri d'intervento in economia (BBC - SWB, 9 aprile 1992); particolare attenzione viene dedicata, tuttavia, alla questione sociale ed ambientale.

Sulla questione nazionale il partito auspicava la costituzione di una federazione tripartita, nell'ambito della quale ogni membro potesse godere di uno status giuridico autonomo (CSTK, n. 4, 1992).

Il problema «nazionale», esploso in Cecoslovacchia con la fine del regime comunista, non ha investito solo la Slovacchia, ma ha fatto la sua comparsa anche nelle regioni ceche.

L'Associazione per la Moravia e la Slesia, nata nell'aprile del 1990, è un movimento politico di centro, espressione degli interessi politici 
ed economici di queste due regioni; obiettivo del partito era la creazione di una federazione tripartita, nella quale la Moravia e la Slesia potessero godere di uno status indipendente (Pehe 1991, n. 10). Alle elezioni del 1992 l'Associazione per la Moravia e la Slesia ha superato la soglia elettorale solo nel parlamento ceco, ottenendo il 5,87\% (Pehe 1992, n. 25).

Tra i partiti di estrema destra solo l'Associazione per la Repubblica/Partito Repubblicano di Cecoslovacchia è riuscito ad avere accesso al parlamento federale con oltre il $6 \%$ dei voti e al parlamento ceco con il 5,98\% dei consensi (BBC - SWB, 11 giugno 1992). Fortemente anticomunista e xenofobo, auspica maggiori poteri per la polizia e la reintroduzione della pena di morte (BBC - SWB, 12 settembre 1991; BBC - SWB, 21 maggio 1992). In campo economico i repubblicani hanno chiesto una minore interferenza dello stato, la fine della «svendita» della proprietà nazionale e maggiori benefici fiscali per le imprese private.

Sul futuro assetto costituzionale i repubblicani auspicavano una Repubblica cecoslovacca unita, con un presidente, un governo con sette ministri e quattro regioni federali: la Boemia, la Moravia-Slesia, la Slovacchia e la SubCarpazia Ucraina (Sladek, BBC - SWB, 20 giugno 1991; BBC - SWB, 2 novembre 1991).

Nei primi mesi del 1992 i partiti Socialista, Verde e Agrario hanno dato vita ad una coalizione elettorale dal nome Unione Liberale Sociale (ULS), che alle ultime elezioni politiche ha ottenuto il $6,52 \%$ dei voti nel parlamento ceco, il 5,84\% nella Camera del Popolo e il $6,06 \%$ dei voti nella Camera delle Nazioni (BBC - SWB, 12 giugno 1992; Pehe 1992, n. 25). L'ULS ritiene indispensabile una riforma economica radicale da realizzarsi, però, in maniera graduale e in modo da salvaguardare i ceti sociali più deboli; a tal fine auspica l'introduzione di sussidi a favore dei giovani, dei pensionati, dei disoccupati e di quanti si trovino in situazioni precarie (CSTK, n. 4, 1992). In agricoltura è per la trasformazione delle cooperative di proprietari collettivi in cooperative di proprietari individuali.

Sul problema dell'ordinamento territoriale era a favore del mantenimento di uno stato comune federale tra cechi e slovacchi.

Il Partito Social-Democratico Cecoslovacco (PSDC), costretto, nel 1948 , ad unirsi al partito comunista al potere, dal quale si è staccato il 27 novembre 1989 , per ricominciare a funzionare come partito politico indipendente (CSTK, n. 1, 1992; Pehe 19 dicembre 1989). La lunga assenza dalla vita politica cecoslovacca è però costata ad esso la perdita della propria base elettorale; molti potenziali elettori di sinistra hanno, infatti, preferito votare per il $F C$, considerato l'autentico 
garante del mutamento politico ed economico. Alle elezioni del 1990 infatti il PSDC non è riuscito a superare la soglia del $5 \%$. La confluenza in esso di alcuni gruppi e deputati di sinistra del $F C$ tuttavia gli ha permesso, in un secondo momento, l'accesso allo stesso parlamento del 1990 (Reiman 1992). La riorganizzazione del partito e l'aumento costante del numero di iscritti (Hajek 10 luglio 1992), hanno consentito al PSDC di passare dal 3,84\% di voti del giugno 1990 a più del $7 \%$ di consensi elettorali alle elezioni del 1992 (BBC - SWB, 12 giugno 1992; Pehe 1992, n. 25). Nel loro programma elettorale i social-democratici si richiamano alle correnti social-democratiche europee; auspicano, infatti, una riforma economica lenta e graduale e l'adozione di misure sociali in difesa dei ceti colpiti dalla transizione (Obrman 1990, n. 23).

Sulla «questione nazionale» il partito si dichiarava favorevole al mantenimento di uno stato comune (CSTK, Daily News and Press Agency, 19 giugno 1992). Di fronte all'evoluzione dei rapporti tra cechi e slovacchi, Dubcek ha affermato tuttavia, in una intervista, che l'eventuale scioglimento della federazione è un problema che riguarda i cittadini cecoslovacchi, che devono essere consultati tramite referendum (Dubcek, BBC - SWB, 27 luglio 1992).

Il Partito Comunista di Boemia e Moravia (PCBM $)^{14}$, nonostante la forte opposizione di cui è stato oggetto, alle elezioni del giugno 1990 ha ottenuto quasi il $14 \%$ di voti, diventando il secondo partito, dopo il Forum Civico, a livello Federale e nella Repubblica Ceca ed il quarto in Slovacchia col 13\% dei voti (Pehe 1990, n. 39). Alle elezioni del 1992, insieme alla Sinistra Democratica, ha dato vita ad una coalizione denominata Blocco di Sinistra, che ha ottenuto più del $14 \%$ dei voti, sia al parlamento federale che a quello ceco. Dal crollo del regime comunista in poi, le modifiche organizzative, la diminuzione degli iscritti, il ricambio della leadership e soprattutto i mutamenti programmatici ed ideologici che hanno investito il partito sono stati notevoli. Anche il partito comunista ha infatti abbracciato un orientamento di tipo riformista, sebbene non propriamente social-democratico; ha accettato il sistema parlamentare multipartitico, la liberalizzazione culturale e ideologica, l'uguaglianza tra le varie forme di proprietà; ma ha sottolineato, al contempo, la sua opposizione verso un ritorno al capitalismo. La riforma economica, secondo il PCBM, deve essere accompagnata da adeguate misure sociali in difesa dei ceti deboli (BBC SWB, 4 ottobre 1991). Tranne che a Praga, dove è più debole, il con-

14 Il Partito comunista cecoslovacco al congresso del partito del 3-4 novembre 1990 ha assunto il nome di Partito comunista di Boemia e Moravia. Cfr. Pehe (1990, $n$. 48). Cfr. anche Keesing's Record of World Events - News Digest for March 1990, p. 37326. 
senso per il $P C B M$ è diffuso in tutta la Repubblica ceca, ed è più forte nelle zone più industrializzate del paese e tra gli elettori più anziani $\mathrm{e}$ di status socio-economico più basso (Obrman 1992, n. 26).

Sulla questione dell'ordinamento territoriale si pronunciava a favore di uno Stato comune costruito su basi volontarie (CSTK, n. 4, 1992).

\section{I partiti dello spettro politico slovacco}

I Cittadini contro la Violenza $(\mathrm{CcV})$, il movimento politico slovacco che, insieme al $F C$, è stato uno dei protagonisti principali della transizione dal regime comunista, ha subito la stessa sorte toccata al $F C$, nonostante godesse di una struttura organizzativa più forte. A partire dalla seconda metà del 1990 , infatti, il movimento $C c V$ ha subito un lento, ma irreversibile processo di disintegrazione, causato soprattutto dalle crescenti divergenze tra «indipendentisti» e «sostenitori della federazione» ${ }^{15}$. I principi fondamentali della sua piattaforma programmatica sono molto simili a quelli del $F C$. Sulla questione territoriale $\mathrm{CcV}$ era a favore del mantenimento della federazione tra due repubbliche con uguali diritti e per la divisione territoriale amministrativa della Slovacchia (BBC - SWB, 6 giugno 1991).

Il 5 marzo del 1991 la fazione guidata dall'ex premier slovacco Vladimir Meciar ${ }^{16}$ si è staccato dal movimento $C c V$, dando vita a un nuovo partito politico, il Movimento per una Slovacchia Democratica, il cui congresso di fondazione si è svolto il 22 giugno 1991 (CSTK, n. 4, 1992). Nell'ottobre dello stesso anno il movimento $C c V$ ha deciso di trasformarsi in partito politico, e al congresso del marzo 1992 ha scelto di chiamarsi Unione Democratica Civica (BBC - SWB, 31 marzo 1992). Questa nuova formazione intende diventare un moderno parti-

15 La scissione verrà formalmente sancita al congresso straordinario tenutosi a Praga il 23 febbraio 1991. Cfr. Keesing's Record of World Events, News Digest for February 1991.

16 Vladimir Meciar è nato nel 1942 a Zvolen, nella Slovacchia Centrale. Ha completato la scuola secondaria nel 1959 e ha lavorato come impiegato al Comitato del distretto nazionale. Nel 1967-'68 fu presidente del Comitato del distretto dell'Unione della Gioventù Cecoslovacca e vice presidente del Comitato di Controllo del Popolo a Ziar nad Hronom. Nel 1970 venne privato delle sue funzioni e della tessera del partito comunista, a causa del suo comportamento al Congresso della Gioventù Slovacca nel 1969. Nel 1970 lavora come fonditore fino al 1973, anno in cui si laureerà alla facoltà di legge dell'Università Comenius e svolgerà la professione di avvocato.

Dopo la rivoluzione di «velluto» del 1989 venne nominato, come esponente del movimento politico Cittadini contro la Violenza, nel gennaio del 1990, Ministro degli Interni e dell'Ambiente slovacco e dopo le elezioni del 1990 premier del governo slovacco, carica che manterrà fino all'aprile del 1991, quando verrà sostituito da Carnogursky. Attualmente occupa, per la seconda volta, la carica di Primo Ministro slovacco. 
to di centro-destra; nel suo programma elettorale viene sottolineata l'importanza di una rapida e ampia privatizzazione della proprietà statale, dell'economia di mercato e della libertà di impresa. Avoca, inoltre, un decentramento del potere e poteri statali limitati.

Alle ultime elezioni politiche, tuttavia, non è riuscita a superare la soglia del $5 \%$.

Il Movimento per una Slovacchia Democratica (MSD) è nato principalmente come tentativo di dare alla Slovacchia uno status politico diverso nell'ambito della federazione. $\grave{E}$, insieme al $P D C$, il vincitore delle elezioni del giugno 1992, avendo conquistato più del $33 \%$ dei voti all'Assemblea Federale, e il $37,26 \%$ al parlamento slovacco (BBC - SWB, 12 giugno 1992; BBC - SWB, 9 giugno 1992).

L'opposizione del partito di Meciar nei riguardi di Praga ha avuto come oggetto la politica economica seguita dal potere centrale e la questione dell'ordinamento territoriale (Pehe 1991, n. 12). Il MSD, pur essendo favorevole alla transizione ad una economia di mercato, intende tuttavia seguire una via o, per meglio dire, tempi differenti rispetto al partito di Klaus. La privatizzazione dell'economia slovacca deve avvenire gradualmente, viste le maggiori difficoltà economiche della regione e lo stato, in queste fasi di transizione, deve svolgere un ruolo di intervento di un certo peso, a difesa dei ceti più colpiti dalla riforma economica (BBC - SWB, 11 gennaio 1992). Alle accuse di affossamento della riforma economica e di ritorno al socialismo, Meciar rispondeva, in una intervista, che quello del suo partito è un programma sociale, che il processo di privatizzazione continuerà, ma che lo stato non intende permettere arricchimenti ingiustificati, o vendite al di sotto dei prezzi reali (Meciar 18 luglio 1992).

Sulla questione nazionale, Meciar, in un'altra intervista rilasciata nel mese di marzo dello stesso anno, ha enunciato gli stadi da seguire per la creazione di un nuovo ordinamento costituzionale: dichiarazione incondizionata di sovranità della Repubblica slovacca e adozione di una costituzion $\mathrm{e}^{17}$; riconoscimento internazionale del nuovo stato e scelta di un presidente come capo dello stato; svolgimento di un referendum per decidere del futuro della federazione (Meciar 26 marzo 1992).

17 Il 17 luglio 1992 il parlamento slovacco ha approvato la dichiarazione di sovranità della Repubblica slovacca, contro la quale hanno votato il Movimento Democratico Cristiano e la Coalizione di partiti Ungheresi. Il 3 settembre del 1992 il Consiglio Nazionale slovacco ha approvato la nuova Costituzione della «nazione slovacca». I deputati ungheresi hanno, anche questa volta, contestato il nuovo documento, e per protesta hanno abbandonato l'aula del parlamento e hanno rifiutato di votare a favore di una costituzione che, secondo loro, li priva dei diritti culturali e politici propri di una minoranza. Cfr. Obrman (1992, n. 31). 
Il Movimento Democratico Cristiano ( $M D C$ ), nato nel febbraio del 1990, ha fatto parte, dopo le elezioni del 1990 , delle coalizioni governative federale e slovacca. Alle elezioni del 1992 ha ottenuto l' $8,88 \%$ al parlamento slovacco e quasi il $9 \%$ dei voti all'Assemblea Federale. Ritiene fondamentale la rigenerazione spirituale della società e appoggia gli obiettivi di fondo della riforma economica cecoslovacca (CSTK, n. 4, 1992). Nel suo programma elettorale il MDC auspica, però, più forti misure sociali tese ad alleviare i problemi causati dalla transizione da una economia pianificata a una di mercato (Carnogursky 11 maggio 1992).

Sul problema dei rapporti tra cechi e slovacchi, il $M D C$ in un primo tempo ha appoggiato l'idea di un ordinamento confederale, allo scopo di garantire una maggiore autonomia alla Slovacchia (Pehe 1992, n. 13), ma nell'estate del 1991, sottoposto a un gran numero di pressioni contrapposte, provenienti sia dal governo federale, che dai partiti nazionalisti slovacchi, ha abbandonato tale idea, optando per la creazione di un'ampia struttura federale. Tale decisione non solo ha provocato, nella seconda metà del 1991, la diminuzione dei suoi consensi, ma ha anche spinto la fazione di centro-sinistra del movimento, guidata da Klepak, a staccarsi dal MDC e dar vita al Movimento Democratico Cristiano Slovacco, sostenitore, quest'ultimo, di una vera e propria autonomia politica ed economica della Slovacchia (Pehe 1992, n. 13; Cannatà 16 aprile 1992) ${ }^{18}$.

Il Partito Comunista slovacco già subito dopo l'introduzione del multipartitismo ha intrapreso un processo di autoriforma mediante un'operazione di ricambio sia della leadership che dei funzionari più screditati e compromessi col vecchio regime (Butora, Butorova e Rosova 1991). In effetti $i$ comunisti slovacchi sono riusciti a crearsi una nuova immagine politica, proponendosi come i veri difensori dei gruppi socialmente più deboli. Il «nuovo» Partito della Sinistra Democratica $(P S D)^{19}$, sotto la direzione di Peter Weiss, eletto presidente al congresso del 20-21 ottobre 1990 (Pehe 1990, n. 48), ha operato una chiara scelta a favore degli ideali social-democratici e nazionalisti che ha contribuito ad accrescere notevolmente i suoi consensi. Alle elezio-

18 Sul problema dell'ordinamento territoriale, il nuovo movimento politico, che non è riuscito a superare la soglia del $5 \%$, ritiene prioritaria la sovranità della Slovacchia, l'adozione di una nuova costituzione e il riconoscimento internazionale della nuova nazione. Cfr. New Faction of Christian Democratic Movement to Field Own Election Candidates, CSTK in English $1342 \mathrm{gmt}, 8$ marzo 1992, in BBC - SWB, 10 marzo 1992. Vedi anche: Radio Czechoslovakia $1700 \mathrm{gmt}, 28$ marzo 1992, in BBC - SWB, 31 marzo 1992.

19 Nell'ottobre del 1990, al congresso del Partito Comunista slovacco, i delegati votarono a maggioranza per il cambiamento del nome, e al congresso di gennaio 1991 la leadership del partito sancì l'avvenuta modifica del nome e l'obbligo di reiscrizione per tutti i membri dell'ex Partito Comunista slovacco. 
ni politiche del giugno 1992 ha ottenuto il 14,70\% dei voti al Consiglio Nazionale slovacco (contro il $13,34 \%$ dei suffragi delle elezioni del 1990) e oltre il $14 \%$ al parlamento federale (Pehe 1992, n. 25). Sostenitore di uno stato democratico, fondato sul diritto e sui principi del welfare, il PSD considera importante il ruolo dello stato in campo economico, e ritiene che esso non possa e non debba rinunciare alle proprie responsabilità nei confronti delle imprese statali; sottolinea, inoltre, l'urgenza di una riforma dei rapporti di proprietà in agricoltura e la conversione delle industrie di armamenti in industrie di pace (CSTK, n. 4, 1992).

Sulla questione dell'ordinamento costituzionale, il partito sottolineava la sostanziale differenza delle proprie politiche da quelle degli altri partiti nazionalisti, quali ad esempio quelle del Movimento per una Slovacchia Democratica. L'obiettivo del PSD era lo sviluppo della Slovacchia come parte di una più ampia federazione tra due repubbliche nazionali eguali e sovrane (BBC - SWB, 18 dicembre 1991).

Un partito fortemente separatista è il Partito Nazionale Slovacco, $(P N S)$, entro il quale è confluito, nel maggio del 1991, il Partito Indipendente degli Slovacchi (BBC - SWB, 5 giugno 1992). Alle elezioni del 1992 ha ottenuto il 7,93\% dei voti al parlamento slovacco e oltre il $9 \%$ dei consensi all'Assemblea Federale.

Il nazionalismo domina l'agenda del partito, come traspare anche da un'intervista rilasciata dal presidente del partito J. Prokes nel giugno del 1991 (Prokes 10 giugno 1991). Su tale issue gli obiettivi principali che il partito intendeva perseguire erano la dichiarazione di indipendenza della Slovacchia, il riconoscimento di quest'ultima come soggetto di diritto internazionale ed il suo ingresso nelle Nazioni Unite (CSTK, n. 4, 1992).

Tra i tanti piccoli partiti che si richiamano ad una singola issue etnica, solo la coalizione composta da: Movimento Democratico Cristiano Ungherese - Coesistenza - Partito del Popolo Ungherese è riuscita ad avere accesso al parlamento federale, con oltre il $7 \%$ dei voti ${ }^{20}$. Rappresentante degli interessi della comunità magiara nella Slovacchia, fa appello ai principi di libertà, di giustizia ed ai valori cristiani; in economia sottolinea l'importanza della creazione di un libero mercato e dello sviluppo economico e sociale delle regioni abitate da gruppi di minoranza (CSTK, n. 1, 1992).

Sulla questione nazionale la coalizione ungherese auspicava il mantenimento dello stato comune nell'ambito di un'organizzazione di tipo federale o provinciale (CSTK, n. 4, 1992).

20 In Cecoslovacchia, ma soprattutto in Slovacchia, la comunità magiara costituisce il gruppo etnico più folto: nella Repubblica ceca circa lo $0,2 \%$ della popolazione è costituito da ungheresi, mentre nella Slovacchia essi raggiungono una percentuale del $10,8 \%$ della popolazione. Cfr. Reisch (1992). 


\section{Riferimenti bibliografici}

Bufalini, J. (1992), Praga al voto con l'incognita slovacca, in «L'Unità», 4 giugno.

Butora, M., Z. Butorova e T. Rosova (1991), The Hard Birth of Democracy in Slovakia: The Eighteen Months Following The "Tender» Revolution, in «The Journal of Communist Studies», VII, n. 4.

Cannatà, F. (1992), Praga, due Slovacchie alle urne di giugno, in «Il Manifesto», 16 aprile.

Cappelli, O. (1990), Comparative Communism's Fall. The First Phase: The «Intelligentsia Revolution» of 1989-1990, in «The Harriman Institute Forum», III, n. 6.

- (1992), La resistibile ascesa della rappresentanza politica in URSS, in «Rivista Italiana di Scienza Politica», XXII, pp. 85-136.

Carnogursky, J. (1992), testo dell'intervista, Slovak Radio, Bratislava $1630 \mathrm{gmt}, 8$ maggio 1992, in «BBC - Summary of World Broadcasts», 11 maggio.

Di Leo, R. (1981), Operai al governo, in «Laboratorio politico», n. 1.

Dubcek, A. (1992), stralci da una intervista, Slovak Radio, Bratislava, $1630 \mathrm{gmt}, 23$ luglio 1992, in «BBC - Summary of World Broadcasts», 27 luglio.

- (1992), stralci da un'intervista, Slovak Radio, Bratislava $1630 \mathrm{gmt}$, 23 luglio 1992, in «BBC - Summary of World Broadcasts», 27 luglio.

Duverger, M. (1961), I partiti politici, Milano, Comunità.

Hajek, M. (1992), Separatismo in Slovacchia? Ma esiste anche fra $i$ ce$c h i$, in «L'Unità», 9 giugno.

- (1992), L'inverno della sinistra praghese stretta tra destra e sinistra, «L'Unità», 10 luglio.

Havel, V. (1992), stralci da una intervista rilasciata il 10 gennaio 1992, Radio Czechoslovakia 1315 gmt, 12 gennaio 92, in «BBC - Summary of World Broadcasts», 14 gennaio.

- (1992), Meditazioni estive, Milano, Feltrinelli.

- (1992), conferenza stampa, estratto dal rapporto di Stanislav Benda dal Castello di Lany, Radio Czechoslovakia 1500 gmt, 20 luglio 1992, in «BBC - Summary of World Broadcasts», 22 luglio.

Hazan, Baruch A. (1985), The East European Political System. Instrument of Power, Boulder e London, Westview Press.

Holmes, L. (1986), Politics in the Communist World, Oxford, Clarendon Press.

Kasabian, K. (1992), The Road to the End... The Rise and Fall of the Velvet Presidency, in «Prognosis», II, luglio-agosto.

Klaus, V. (1992), Dismantling Socialism - Preliminary Report (a Road to Market Economy II), Praha, Top Agency.

Kroupa, D. (1992), intervista a cura di V. Mlynar, in «Prognosis», n. 6. 
Lijphart, A. (1984), Le democrazie contemporanee, Bologna, Il Mulino. Lipset, S.M. e S. Rokkan (1967), Cleavages Structures, Party Systems, and Voter Alignments: An Introduction, in S.M. Lipset e S. Rokkan (a cura di), Party Systems and Voter Alignments: Cross National Perspective, New York, The Free Press, pp. 1-64.

Lovenduski, J. e J. Woodall (1987), Politics and Society in Eastern Europe, London, MacMillan Education.

Martin, P. (1990), The Election Campaign, in «Radio Free Europe» (RFE), I, n. 24.

Meciar, V. (1992), stralci da un'intervista registrata, Slovak Radio, Bratislava 1630 gmt, 15 luglio 1992, in «BBC - Summary of World Broadcasts», 18 luglio.

- (1992), testo dell'intervista, Slovak Radio, Bratislava 1730 gmt, 22 marzo 1992, in «BBC - Summary of World Broadcasts», 26 marzo.

- (1992), Slovaks Air their Gripes, in «The Prague Post», II, n. 31.

Mitchell, M. (1992), Leaders Propose Blueprint for Nation's Breakup, in «The Prague Post», II, n. 30.

Morlino, L. (1986a), Democrazie, in G. Pasquino (a cura di), Manuale di scienza della politica, Bologna, Il Mulino, pp. 83-135.

- (1986b), Consolidamento Democratico: definizioni e modelli, in «Rivista Italiana di Scienza Politica», XVI.

- (1992), Partiti e consolidamento democratico nel Sud Europa, in M. Calise (a cura di), Come cambiano i partiti, Bologna, Il Mulino.

Obrman, J. (1990), The Main Contenders in the Elections, in «RFE», I, n. 23.

- (1992), President Havel's Diminishing Political Influence, in «Radio Free Europe/Radio Liberty» (RFE/RL), I, n. 11.

- (1992), The Czechoslovak Elections, in «RFE/RL», I, n. 26.

- (1992), Slovakia Declares Sovereignty; President Havel Resign, in «RFE/RL», I, n. 31.

- (1992), Czechoslovakia: a Messy Divorce after all, in «RFE/RL», I, n. 41.

Pehe, J. (1989), Political Changes Follow Unrest, in «RFE», 19 dicembre.

- (1989), The Civic Forum and the Public Against Violence, in «RFE», 19 dicembre.

- (1990), An Overview of the Democratic Revolution, in «RFE», I, n. 10.

- (1990), The Postrevolutionary Federal Assembly Holds Its last Session, in «RFE», I, n. 22.

- (1990), Civic Forum and Public Against Violence after the Elections, in «RFE», I, n. 25.

- (1990), The Communist Party at a Crossroads, in «RFE», I, n. 39.

- (1990), Party System Struggles to Develop, in «RFE», I, n. 43.

- (1990), The Civic Forum Shift to the Right, in «RFE», I, n. 47. 
- (1990), Changes in the Communist Party, in «RFE», I, n. 48.

- (1991), The Agenda for 1991, in «RFE», II, n. 3.

- (1991), The Civic Forum Becomes a Political Party, in «RFE», II, n. 5.

- (1991), The First Weeks of 1991: Problems Solved, Difficulties Ahead, in «RFE», II, n. 10.

- (1991), The Civic Forum Splits into Two Groups, in «RFE», II, n. 10.

- (1991), Growing Slovak Demands Seen as Threat to Federation, in «RFE», II, n. 12.

- (1991), The Realignment of Political Forces, in «RFE», II, n. 21.

- (1992), Czechoslovakia's Changing Political Spectrum, in «RFE/ RL», I, n. 5.

- (1992), Czechoslovak Federal Assembly Adopts Electoral Law, in «RFE/RL» Research Report, I, n. 7.

- (1992), Slovak Nationalism Splits Christian Democratic Ranks, in «RFE/RL», I, n. 13.

- (1992), Czechoslovakia's Political Balance Sheet, 1990 to 1992, in «RFE/RL», I, n. 25.

- (1992), Czech and Slovaks Define Postdivorce Relations, in «RFE/ RL», I, n. 45.

- (1992), Czechoslovak Parliament Votes to Dissolve Federation, in «RFE/RL», I, n. 48.

Pitkin, H.F. (1967), The Concept of Representation, Berkeley, University of California Press.

Pravda, A. (1986), Elections in Communist Party States, in S. White e D. Nelson (a cura di), Communist Politics. A Reader, London, MacMillan Education.

Pridham, G. (1991), Democratic Consolidation in Theory and Practice: Southern European Lessons for Eastern Europe?, relazione presentata alla Tavola Rotonda su «Transition to Democracy: Eastern Europe in comparative perspective», European Consortium for Political Research (ECPR), Research Sessions, Forlì, 25 ottobre.

Prokes, J. (1991), estratto da una intervista, Bratislava 1000 gmt, 6 giugno 1991, in «BBC - Summary of World Broadcasts», 10 giugno.

Reimann, M. (1992), Dopo la rivoluzione. Il futuro incerto della Cecoslovacchia alla vigilia delle elezioni, in «Il Passaggio», V, n. 2.

Reisch, A. (1992), Hungarian Ethnic Parties Prepare for Czechoslovak Elections, in «RFE/RL», I, n. 18.

Riosa, A. (1974), Storia dei paesi dell'Europa Orientale, Milano, CELUC.

Sartori, G. (1976), Parties and Party Systems. A Framework for Analysis, Cambridge, Cambridge University Press.

- (1990), I sistemi elettorali, in G. Sartori, Elementi di teoria politica, Bologna, Il Mulino. 
Sladek, M. (1991), testo dell'intervista, Bratislava, 1000 gmt, 17 giugno 1991, in «BBC - Summary of World Broadcasts», 20 giugno.

Ulc, O. (1982), Legislative Politics in Czechoslovakia, in D. Nelson e $\mathrm{S}$. White (a cura di), Communist Legislatures in Comparative Perspective, London, MacMillan Press.

Von Beyme, K. (1992), L'europeizzazione dell'Europa orientale, in M. Calise (a cura di), Come cambiano i partiti, Bologna, Il Mulino.

Weiss, P. (1991), Democratic Left Leader on his Party's Role in Slovak Opposition, testo dell'intervista con P. Weiss, in «BBC - Summary of World Broadcasts», 13 giugno.

Wightman, G. (1991), The Collapse of Communist Rule in Czechoslovakia and the June 1990 Parliamentary Elections, in «Parliamentary Affairs», XLIV, n. 1. 